\section{Working Memory, Processing Speed, and IQ in Youth with Mood Disorders and Psychotic Features}

\section{Abstract}

The presence of psychotic symptoms is relatively common in severe manifestations of Bipolar Disorder (BD) and Major Depressive Disorder (MDD), but their occurrence and their effect on cognitive functioning in children and adolescents remain under-investigated areas of research. This brief report summarizes a study with child and adolescent psychiatric inpatients indicating that the presence of psychotic features with pediatric mood disorders adversely affects working memory and overall cognitive functioning ability.

Keywords: Bipolar disorder; Cognitive deficits; Major depressive disorder; Psychosis

Received: November 14, 2016; Accepted: January 06, 2017; Published: January 16, 2017

\section{Introduction}

The presence of psychotic symptoms with mood disorders, such as Major Depressive Disorder (MDD) or Bipolar Disorder (BD), in children and adolescents seems to reflect severe manifestations of the disorders [1]. Research with adults with MDD and BD has generally revealed concomitant impairments in attention, processing speed, working memory, and executive functioning deficits $[2,3]$. Cognitive deficits in children and adolescents with severe mood disorders and psychosis have been less thoroughly examined [4-6].

\section{Methods}

In a study of the cognitive functioning of discharged child and adolescent psychiatric inpatients, we investigated the WISCIV Full Scale IQ scores that were obtained following admission, and the working memory and processing speed of 80 children and youth, aged 8-17 years, with mood disorders, with and without accompanying psychotic features. Working memory was assessed by the WISC-IV digit span subtest, and processing speed was assessed via the Symbol Search and Coding Subtests. The psychiatric diagnosis on admission was utilized to identify diagnostic categories as well as the presence or absence of psychotic features. Based on DSM-IV criteria, the diagnostic groups included MDD (7 patients), BD (43 patients), Mood Disorder, Not Otherwise Specified (NOS) (30 patients). A total of 8 patients with MDD or BD had psychotic symptoms. No additional $(\mathrm{SD}=2.47)$.

\section{Results}

\section{James B McCarthy', Kristin T Segovitch ${ }^{2}$, Shira R Weiss ${ }^{3}$ and Baptiste Barbot ${ }^{4}$}

\author{
1 New York City Campus, Pace University, \\ NY 10038, USA \\ 2 New York City Children's Center, Queens \\ Campus, USA \\ 3 Sagamore Children's Psychiatric Center, \\ NY, USA \\ 4 Child Study Center, Yale University, NY \\ 10038, USA
}

Corresponding author:

James B McCarthy

झ jmccarthy@pace.edu

New York City Campus, Pace University, 41 Park Row, NY 10038, USA.

Tel: 212-346-1796

Citation: McCarthy JB, Segovitch KT, Weiss $\mathrm{SR}$, et al. Working Memory, Processing Speed, and IQ in Youth with Mood Disorders and Psychotic Features. J Child Dev Disord. 2017, 3:1.

symptom inventory or severity scales were utilized. There were 43 females and 37 males, and the mean age was 14.21 years

Sample size was deemed acceptable for planned analysis based on a priori power analysis ( $d=0.2, a=0.05$ ) suggesting a sample size of 63 subjects. Differences in diagnosis and the presence or absence of psychotic features were generally unrelated to sex $(p=082)$, race $(p=081)$, and age although the patients with MD, (NOS) were slightly younger than the BD and MDD groups $(p<0.001)$. Controlling for sex, age, and presence of psychotic symptoms, a Multivariate Analysis of Variance indicated no significant differences in cognitive functioning between the MDD and BD 
groups $(F[8,124]=0.46, \mathrm{p}=0.88)$, or between those with MDD or $\mathrm{BD}$ (combined) and those with MD, NOS $(F[4,64]=0.06, \mathrm{p}=0.99)$. The patients with MDD or BD and psychotic symptoms showed lower Full Scale IQ scores $\left(F[2,66]=3.50, p=0.036, \mathrm{n}^{2}=0.10\right)$ and worse working memory $\left(F[2,66]=5.19, \mathrm{p}<0.008, \mathrm{n}^{2}=0.14\right)$ than those with MDD or BD without psychotic symptoms, even after the Bonferroni adjustment (adjusted risk $a=0.013$ ). Despite their low general cognitive ability, the patients with MDD or BD in addition to psychotic symptoms didn't demonstrate significantly lower levels of Coding $(p=0.35)$ and Symbol Search $(p=0.47)$ scores than the other groups.

\section{Conclusion}

The results point to the importance of psychotic features associated with severe mood disorders in children and adolescents, as well as the need for additional research on developmental differences in mood disorders and cognition. If the findings of lower Full Scale IQ scores and working memory are replicated in larger studies of children and adolescents with severe mood disorders that utilize comprehensive neuropsychological assessments, the deleterious effect of cognitive deficits will need to be considered in supportive services and treatment interventions. 


\section{References}

1 McCarthy JB, Dobroshi Z (2014) Major depression, bipolar disorder and psychosis in children. J Infant Child Adolesc Psychother 13: 246-261.

2 Godard J, Baruch P, Grondin S, Lafleur MF (2012) Psychosocial and neurocognitive functioning in unipolar and bipolar depression: a 12-month prospective study. Psychiatry Res 196: 145-153.

3 Hill SK, Reilly JL, Harris MS, Rosen C, Marvin RW, et al. (2009) A comparison of neuropsychological dysfunction in first-episode psychosis patients with unipolar depression, bipolar disorder and schizophrenia. Schizophr Res 113: 167-175.
4 Wagner S, Müller C, Helmreich I, Huss M, Tadić A (2015) A metaanalysis of cognitive functions in children and adolescents with major depressive disorder. Eur Child Adoles Psy 24: 5-19.

5 Klimkeit EJ, Tonge B, Bradshaw JL, Melvin GA, Gould K (2011) Neuropsychological deficits in adolescents with unipolar depression. Arch Clin Neuropsychol 26: 662-676.

6 Bilginer L, DeLuca V, Pogge DL, Stokes JS, Harvey PD (2005) Intellectual functioning in adolescents with indicators of psychosis: evidence for decline in functioning related to number of psychotic features. J Neuropsychiatry Clin Neurosci 17: 106-113. 